\title{
Incidence de rejets ponctuels et diffus sur les communautés d'inver- tébrés benthiques d'un cours d'eau du Beaujolais : l'Ardières
}

\author{
B. Faessel 1 \\ M.C! Roger ${ }^{1}$ \\ B. Cazin²
}

Mots clés : Cours d'eau, pollution diffuse, toxiques, macroinvertébrés.

Près de la moitié du bassin versant de l'Ardières (affluent de la Saône) est occupée par la culture de la vigne induisant dans le cours d'eau une pollution diffuse par les eaux de ruissellement.

L'étude a pour but de déterminer la qualité biologique de cette rivière et plus spécifiquement de caractériser l'impact des traitements viticoles (herbicides, fongicides, insecticides) sur les peuplements benthiques.

Quatre stations d'étude sont réparties sur le cours d'eau en fonction des sources de pollution : une station de référence : station 1, une station hors vignoble mais à l'aval d'un effluent de papeterie : station 2 et deux stations dans la zone viticole à l'aval d'affluents traversant également le vignoble : stations 3 et 4 .

La richesse taxonomique et les indices biologiques diminuent fortement dans la zone aval soumise aux pollutions diffuses.

La structure des peuplements d'invertébrés (98 taxons) a également été étudiée au moyen d'une analyse factorielle des correspondances et d'une classification ascendante hiérarchique.

Quatre groupements faunistiques ont ainsi pu être distingués :

- le premier (A) renferme les taxons rencontrés sur le cours supérieur de l'Ardières (stations 1 et 2) dont les effectifs diminuent sur la station 2 (Heptageniidae, Elmidae, Gammaridae...);

- le deuxième (B) comprend les taxons propres à la station de référence. Des taxons permettent de définir le niveau typologique du secteur (B3 : Baetis alpinus, Drusus annulatus, Rhyacophila tristis). D'autres très sensibles à la pollution (Epeorus torrentium, Ecdyonurus sp., Allogamus auricollis, Micrasema moestum...) soulignent la bonne qualité biologique de ce secteur;

- le troisième $(\mathrm{C})$ englobe les taxons inféodés à la station 2, confirmant le caractère organique dominant de la perturbation de ce secteur (Halesus radiatus, Habrophlebia sp., Dasyhelea...) ;

- le quatrième (D) est représenté par les taxons communs à tout le cours d'eau (Hydropsyche angustipennis, Baetis rhodani, Baetis fuscatus, Ephemerella ignita, Simuliidae, Chironomidae...).

L'étude de la structure des peuplements à l'aide de courbes richesse spécifique-abondance permet une caractérisation des effets des différents types de pollution rencontrés sur les biocénoses en place.

Effects of point and nonpoint pollution on benthic invertebrate communities in the river Ardières (Beaujolais)

Keywords : Running water, non-point pollution, toxics, macroinvertebrates.

This study has been carried out along the Ardières. The lower part of this river runs across a wine growing area where chemicals (herbicides, fungicides, insecticides) are intensively used and have caused a non-point pollution in the river.

The purposes of this investigation are :

1) to assess the quality of the river,

2) to evaluate the effect of the toxic pollutants on macroinvertebrate communities and the response of some species to these toxins.

The macrobenthos was collected at four stations from April to December 1991.

The biotic index and the species richness are very low in the polluted lower part of the river.

1. CEMAGREF, Division Biologie des Ecosystèmes Aquatiques, Laboratoire Diagnose des Systèmes Aquatiques, 3 bis quai Chauveau, 69336 Lyon Cedex 09, France.

2. Conseil Supérieur de la Pêche. Laboratoire mobile du CEMAGREF Lyon, France.

Texte d'une communication effectuée dans le cadre du Colloque - Limmologie appliquée et application de la Limnologie - Besançon, Franche-Comté, 16-19 Novembre 1992. 
Correspondance Factorial Analysis applied to the 98 species recorded and a ascending hierarchical classification indicate four groups of species.

- The first group is composed of taxa living upstream (station 1 and 2) ; they are less abundant at the second station (Heptageniidae, Elmidae, Gammaridae).

- The species of the second group are restricted to the first station (Drusus annulatus, Baetis alpinus, Rhyacophila tristis,...).

- The third group includes species from the second station, many of them are generally tolerant of organic matter (Halesus radiatus, Habrophlebia sp... Dasyhelea,...).

- The last group is represented by the most euryecious species, that are living in the whole river (Hydropsyche angustipennis, Baetis rhodani,. Baetis fuscatus, Ephemerella ignita, Simuliidae, Chironomidae...).

\section{Introduction}

Les activités humaines entraînent dans les milieux dulcicoles des perturbations qui peuvent être de type organique, chimique, toxique ou physique.

Ces différentes agressions peuvent avoir sur les biocénoses aquatiques des effets particuliers et/ou caractéristiques. Cependant, les dégradations observées actuellement résultent le plus souvent de pollutions mixtes.

Les pollutions de type organique ont été largement étudiées et leurs effets sont relativement bien connus. De nombreuses méthodes d'évaluation de la qualité des milieux ont été mises au point (voir à ce sujet l'analyse de Washingtón (1984).

En revanche, les effets des pollutions toxiques (micropolluants, métaux lourds, pesticides) sur les biocénoses sont encore mal connus (Kimbal \& Levin 1985). Ils ont essentiellement donné lieu à des études de toxicité sur des organismes tests en conditions expérimentales, afin d'établir des critères de toxicité des produits, dans un souci de règlementation et de normalisation.

L'étude en laboratoire des effets des produits toxiques sur une espèce est utile mais elle n'est pas pertinente pour évaluer les risques encourus par les peuplements en place.

Ainsi, des recherches visant à établir un lien entre les expériences de laboratoire et le milieu naturel se sont développées. L'utilisation d'écosystèmes expérimentaux : mésocosmes (Odum 1984) et travaux de Chapman $(1989,1991)$ ont permis de maîtriser certains des paramètres qui régulent les populations dans leur milieu (composantes morphodynamiques et chimiques). Ce type de recherche peut permettre de préciser les réactions des communautés benthiques (Clements et al. 1988).
Dans le milieu naturel, d'autres facteurs tels que la nutrition, les exigences physiologiques, la prédation, la compétition,... entrent en jeu et conditionnent également la structure des biocénoses. C'est pourquoi il est indispensable de réaliser des observations in situ afin de mesurer l'impact réel des différents polluants.

Seuls quelques travaux portent sur l'étude de l'impact de produits toxiques sur les macroinvertébrés benthiques en milieu naturel (Leaner et al. 1971, Winner et al. 1980, Elouard \& Jestin 1982, Lapoint et al. 1984, Wallace et al. 1991).

Ces auteurs observent généralement une diminution de l'abondance et de la richesse spécifique. La sensibilité ou la résistance de certains taxons ou groupes faunistiques à des polluants, ont, d'autre part, pu être mises en évidence. En particulier, la famille des Chironomidae reste souvent le groupe dominant après ce type de pollution (Winner et al. op. cit.).

Le bassin versant de l'Ardières est essentiellement occupé par la culture de la vigne induisant dans le cour d'eau une pollution diffuse par les eaux de ruissellement.

De nombreux travaux (Roulier 1988, Rapport Cemagref 1988, Nore 1988, Munoz \& Belamie 1990) sur la nature, la quantité et le devenir des différents produits de traitement utilisés ont été réalisés sur ce bassin versant. Dans ce contexte, il est intéressant de connaître l'impact de ces produits sur la faune de ce cours d'eau.

La présente étude a pour objet d'établir un diagnostic de l'état de l'Ardières et d'en déterminer la qualité biologique. Un second objectif est de caractériser de façon plus spécifique l'impact, sur les macroinvertébrés benthiques, des substances utilisées en viticulture et d'en apprécier le degré de toxicité potentiel. 


\section{Présentation du milieu}

L'Ardières, affluent de la rive droite de la Saône, draine un bassin versant de $220 \mathrm{~km}^{2}$ situé dans le Haut Beaujolais (Fig. 1). Ce terrain est limité au nord par les monts du Maconnais et au sud par le Beaujolais méridional. Cette rivière prend sa source à $850 \mathrm{~m}$ d'altitude au pied du col du Patoux. Elle atteint la cote $170 \mathrm{~m}$ à sa confluence avec la Saône, après un parcours de $28 \mathrm{~km}$.

Le bassin versant est constitué de formations cristallines plus ou moins altérées.

Dans son cours supérieur, le substrat du cours d'eau est composé d'éléments grossiers : pierres grossières et pierres fines selon la classification de Malavoi (Malavoi \& Souchon 1989), reposant sur un substratum sableux qui devient dominant dans la partie aval.

L'activité économique du bassin de l'Ardières est surtout de nature viticole : $\mathbf{4 4} \%$ de la surface plantée en vigne. Le reste des terrains est occupé par des prairies et des forêts (Nore 1988).

De nombreuses études (Collet 1987), Nore 1988, Munoz \& Belamie 1990) ont montré l'existence d'une pollution diffuse liée aux traitements de la vigne : dans l'Ardières à partir de l'aval de Beaujeu et dans ses deux principaux affluents (la Morcille et le ruisseau des Samsons).

L'Ardières reçoit les effluents des stations d'épuration des principales agglomérations qui jalonnent son cours. Les autres sources de pollution sont en amont de Beaujeu : les effluents de la papeterie du Val d'Ardières et ceux de la carrière de Creuzeval par l'intermédiaire du ruisseau de St Didier.

Les résultats d'enquêtes (Collet 1987) ont permis d'obtenir des informations sur la nature des substances utilisées en viticulture, leur qualité et les périodes de traitements. Une cinquantaine de produits ont ainsi pu être identifiés dont 20 fongicides, 10 herbicides et 20 insecticides (Munoz \& Belamie 1990). Ces produits sont partiellement entraînés par lessivage vers les milieux aquatiques.

Ces transferts de pesticides présentent un caractère saisonnier. Les herbicices sont épandus de février à juin alors que les fongicides et les insecticides sont utilisés d'avril à août (Collet 1987). Les contaminations peuvent se produire hors périodes

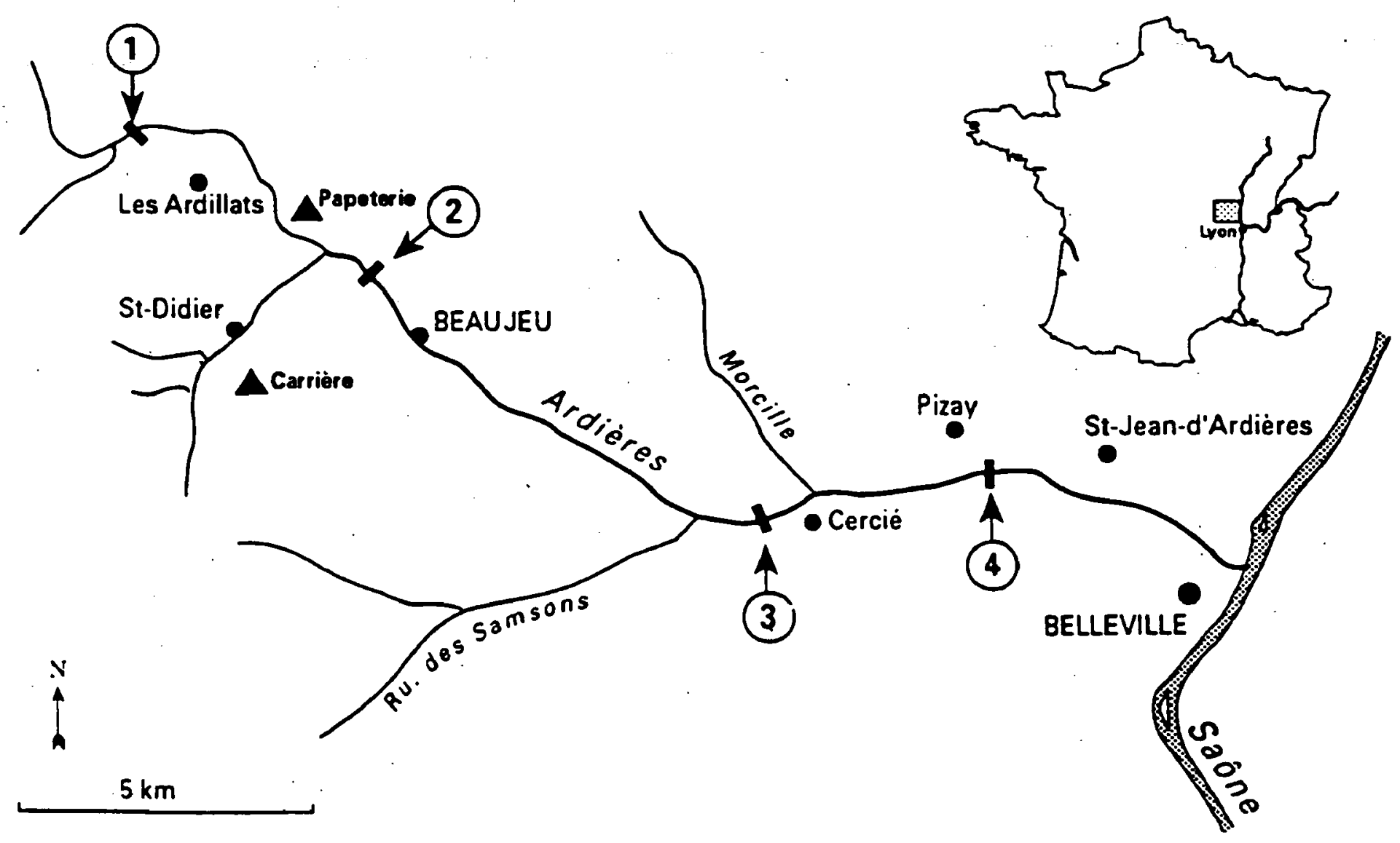

Fig. 1. Situation des stations d'étude.

Fig. 1. Location of the sampling sites. 
de traitements sous forme particulaire ou soluble, lors d'épisodes pluvieux particulièrement importants (Munoz \& Belamie 1990).

\section{Matériel, méthodes et choix des stations}

Le choix des stations a été conditionné par les sources de contamination. Quatre stations ont été retenues (Fig. 1) :

Station 1 : station de référence, située à l'amont des Ardillats, quelques centaines de mètres à l'amont de l'étang Le Perroud ;

Station 2 : amont Beaujeu, au niveau du stade (aval papeterie) ;

Station 3 : le Pré du Bief : aval confluence du ruisseau des Samsons ;

Station 4 : Pont de Pizay, aval confluence de la Morcille.

Les principaux paramètres physiques des stations figurent dans le tableau I.

Les stations 3 et 4 sont fortement soumises aux effets des traitements, le vignoble étant dominant sur cette partie du bassin versant (arrivée du ruisseau des Samsons et de la Morcille). La station 2 semble moins touchée par ce type de pollution, le vignoble ne représentant qu'une faible surface du bassin versant surtout occupé par des prairies et des forêts.

L'étude a été réalisée en 1991 lors de quatre campagnes d'échantillonnage en avril, mai, juillet et décembre.

Les prélèvements sont effectués dans des habitats comparables d'une station à l'autre, en fonction du couple vitesse de courant/granulométrie, une surface totale de $1 \mathrm{~m}^{2}$ étant prélevée sur chaque station :

- 5 échantillons de $0,1 \mathrm{~m}^{2}$ à l'aide d'un filet de type Surber en faciès lotique,

- 1 échantillon de $0.5 \mathrm{~m}^{2}$ à l'aide d'un troubleau en faciès lénitique.

Au laboratoire, les organismes sont extraits après filtration sur une colonne de tamis dont l'ouverture de maille inférieure est de $630 \mu$. Les différents groupes faunistiques ${ }^{1}$ sont ensuite identifiés jusqu'au niveau de l'espèce chaque fois que cela est possible. Les densités de chaque unité taxonomique sont exprimées en classes d'abondance définies par Frontier \& Pichod-Viale (1991).

1. Sauf oligochètes, traités par ailleurs et Chironomidae, difficilement déterminables.

Tableau 1. Principales caractéristiques des stations.

Table 1. Typical characteristics of the stations.

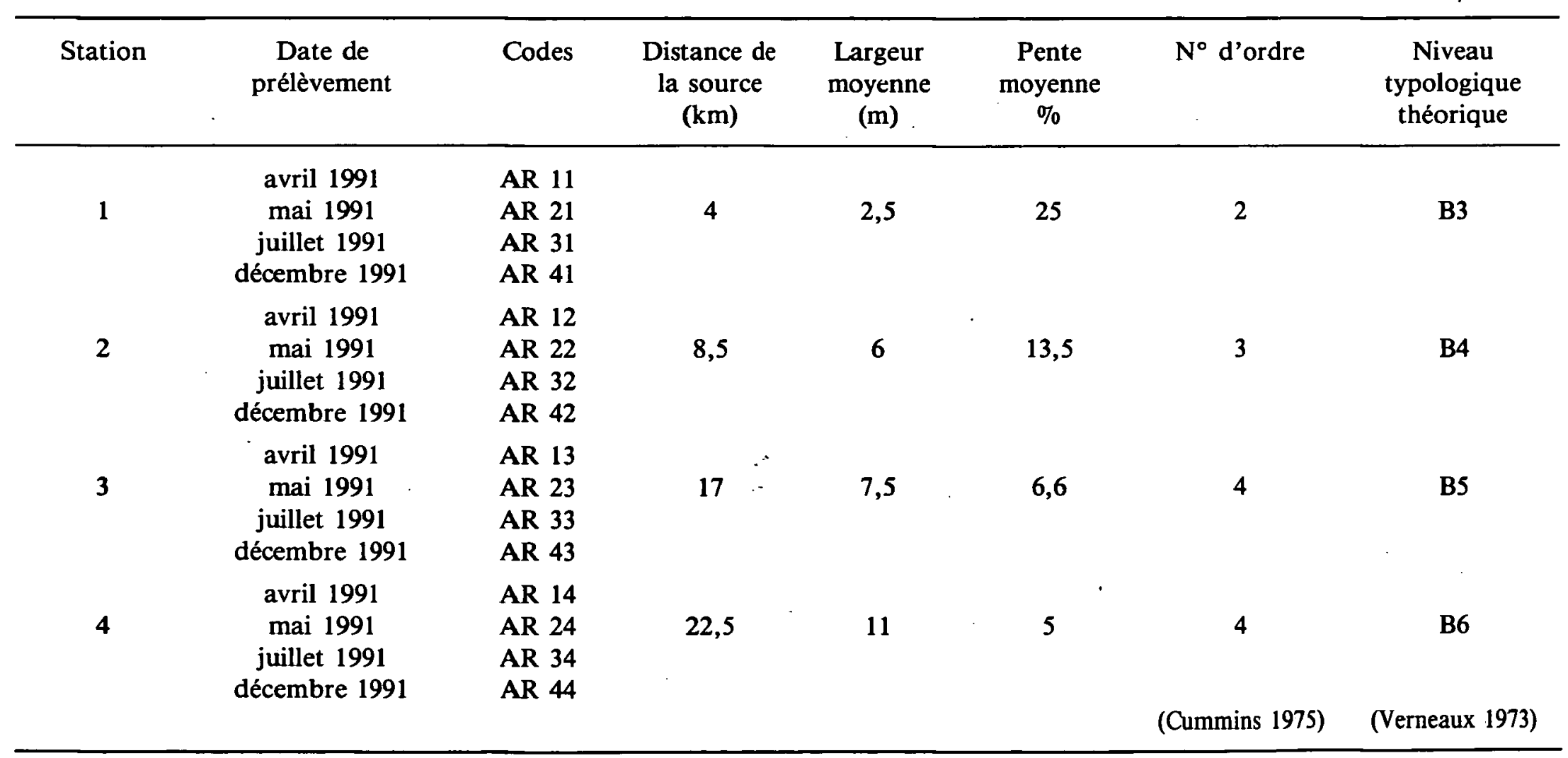




\section{Résultats}

\subsection{Données physico-chimiques}

En avril, juillet et décembre des prélèvements d'eau ont été effectués aux fins d'analyses physicochimiques. Les résultats figurent dans le tableau 2. Des sédiments ont été prélevés en juillet sur toutes les stations et la concentration des six principaux métaux a été mesurée (Tableau 3).

La température maximale relevée en juillet ne dépasse pas $24^{\circ} \mathrm{C}$ sur la station la plus aval. Dans son ensemble ce cours d'eau présente des eaux relativement fraîches; compatibles avec l'existence d'un. peuplement salmonicole. Les teneurs instantanées en $\mathrm{O}_{2}$ dissous traduisent un milieu bien oxygéné.

Les teneurs en matières organiques, mesurées par dosage du Carbone Organique Total (C.O.T.), indiquent, notamment, en avril, une eau de qualité médiocre sur l'ensemble du cours (fortes teneurs en Carbone).

Des concentrations notables en composés minéraux de l'azote et du phosphore sont rencontrées dans l'Ardières. Elles traduisent l'existence d'apports exogènes, liés aux activités humaines. Les teneurs en nitrates, comprises entre 5 et $11 \mathrm{mg} . \mathrm{l}^{-1}$ de $\mathrm{NO}_{3}{ }^{-}$, bien que considérées comme normales, ne sont en aucun cas naturelles.

Des concentrations élevées en nitrites et en sels ammoniacaux sont mesurées aux points 2 et 3 au cours de la campagne de juillet. Les teneurs en phosphates, fortes en été, traduisent un état de pollution sur tous les points d'étude (Nisbet \& Verneaux 1970). L'Ardières présente des eaux peu productives sur le plan piscicole (Cazin 1987). La station 2 apparaît comme la plus dégradée en raison de la concentration élevée en $\mathrm{NH}_{4}{ }^{+}$.

Tableau 2. Résultats des analyses physico-chimiques réalisées sur l'Ardières.

Table 2. Physico-chemical data.

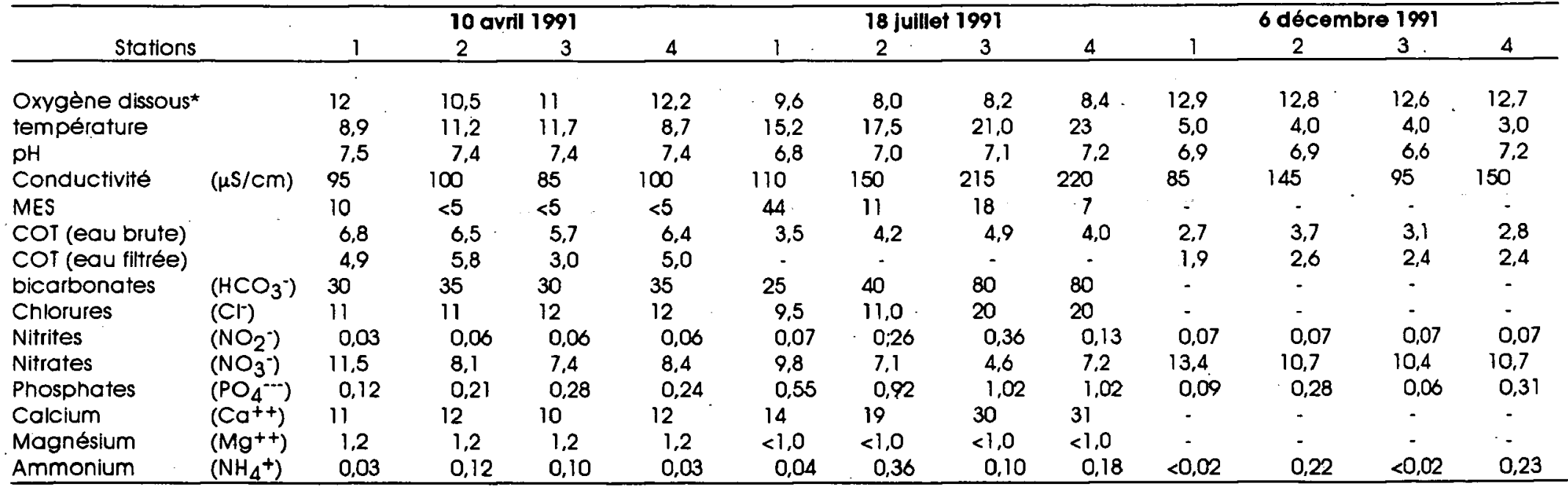

*Concentrations exprimées en $\mathrm{mg} . \mathrm{l}^{-1}$

Tableau 3. Teneur des sédiments de l'Ardières en métaux lourds, juillet 1991. Analyse effectuée par J.L. Roulier, Division Qualité des Eaux.

Table 3. Concentration of heavy metals in sediment, July 1991.

\begin{tabular}{lccccc}
\hline & & $\begin{array}{c}\text { Concentration dans } \\
\text { la phase solide } \\
\text { en } \mathrm{mg} / \mathrm{kg} \text { de poids sec }\end{array}$ & & $\begin{array}{c}\text { Concentration de } \\
\text { référence milieu naturel } \\
\text { (Agence Rhin-Meuse) }\end{array}$ \\
\hline Cadmium & $<2$ & $<2$ & $<2$ & $<2$ & 0,50 \\
Chrome & 11 & 24 & 42 & 34 & 20 \\
Cüivire & 9,7 & 101 & 218 & 187 & 15 \\
Plomb & 34 & 172 & 115 & 94 & 20 \\
Zinc & 70 & 156 & 152 & 122 & 75 \\
Mercure & 0 & 0,08 & 0,20 & 0,08 & 0,10 \\
\hline
\end{tabular}


Selon les critères de l'Agence Rhin-Meuse (Agence Rhin-Meuse, comm. pers.), sur les 6 métaux analysés, seul le cuivre présente des teneurs anormalement élevées aux 3 stations aval. Ces teneurs atteignent le niveau de contamination 2 (entre 5 et 10 fois la concentration de référence) sur la station 2 et. le niveau 3 (plus de 10 fois la valeur de référence) sur les stations 3 et 4 ). Le plomb présente le niveau de contamination 2 sur les stations 2 et 3 .

\subsection{Données biologiques}

\section{- Indices}

Deux types d'indices ont été retenus pour évaluer la qualité biologique de ce cours d'eau : les indices biotiques (Tuffery \& Verneaux 1967) et l'indice biologique de qualité générale (Verneaux et al. 1982).

Les deux méthodes aboutissent à des résultats comparables (Tableau 4) pour l'ensemble des points d'étude aux différentes campagnes d'échantillonnage. L'Ardières se divise en deux secteurs : d'une part, les stations 1 et 2 de qualité biologique correcte avec cependant une légère altération du milieu en juillet et, d'autre part, une zone en aval (stations 3 et 4) fortement dégradée.

\section{- Evolution de la richesse spécifique totale}

L'examen de la richesse spécifique sur chacune des stations confirme l'existence des deux secteurs définis en fonction des indices (Fig. 2) : une partie amont où le nombre de taxons est élevé, et une zone aval (station 3,4) caractérisée par une réduction importante de la richesse taxonomique.

De même, si l'on considère l'évolution saisonnière de cette richesse spécifique, le secteur amont se distingue par une plus forte variabilité du nombre de taxons, celui-ci étant le plus faible en juillet.

\section{- Evolution des principaux groupes faunistiques}

Huit espèces de Plécoptères ont été récoltées. Elles se développent essentiellement sur la station de référence où une vingtaine d'individus appartenant à 5 espèces ont été prélevés au printemps. La station 2 n'héberge plus que deux taxons : quelques Nemouridae et le genre Isoperla ( 3 individus) qui représentent les Plécoptères les plus résistants à une forte teneur en matières organiques (Verneaux 1973).

Les Trichoptères constituent un ordre d'insectes bien diversifié avec 26 et 17 espèces répertoriées dans les 2 stations amont. Sur ce secteur, d'importantes variations des effectifs et de la richesse taxonomique

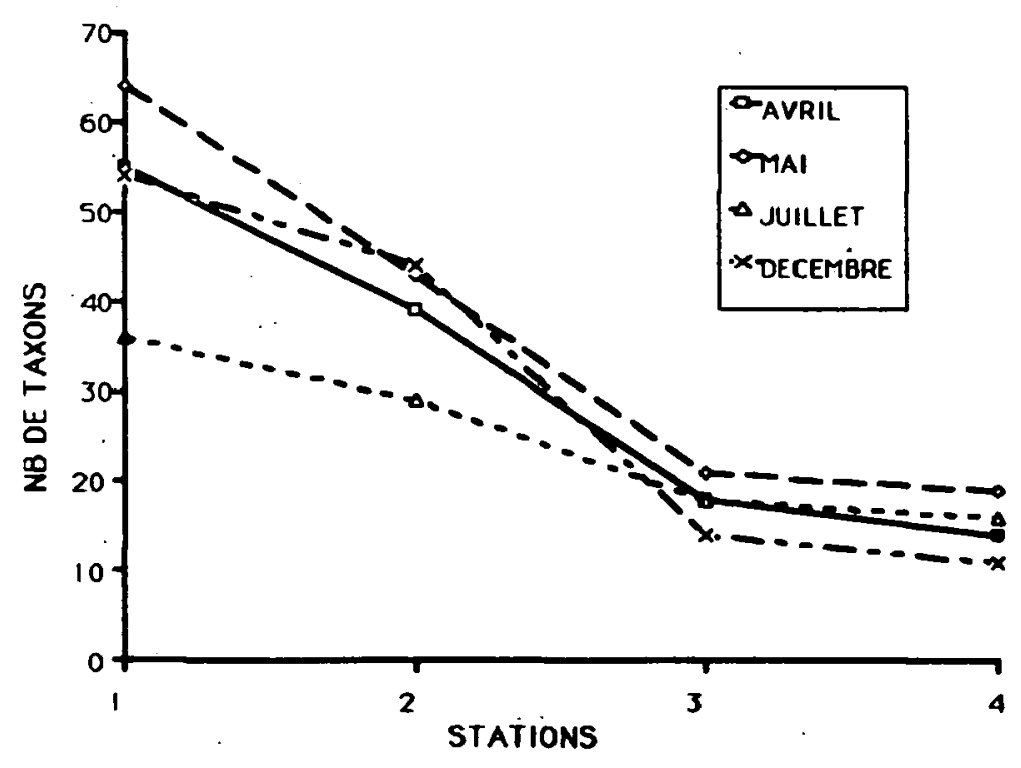

Fig. 2. Evolution de la richesse spécifique totale.

Fig. 2. Change in the total species richness.

Tableau 4. Indices biologiques. $C$ : faciès lotique ; $\mathrm{L}:$ faciès lentique.

Table 4 : Biotic indices. $C$ : lotic facies ; L : lentic facies.

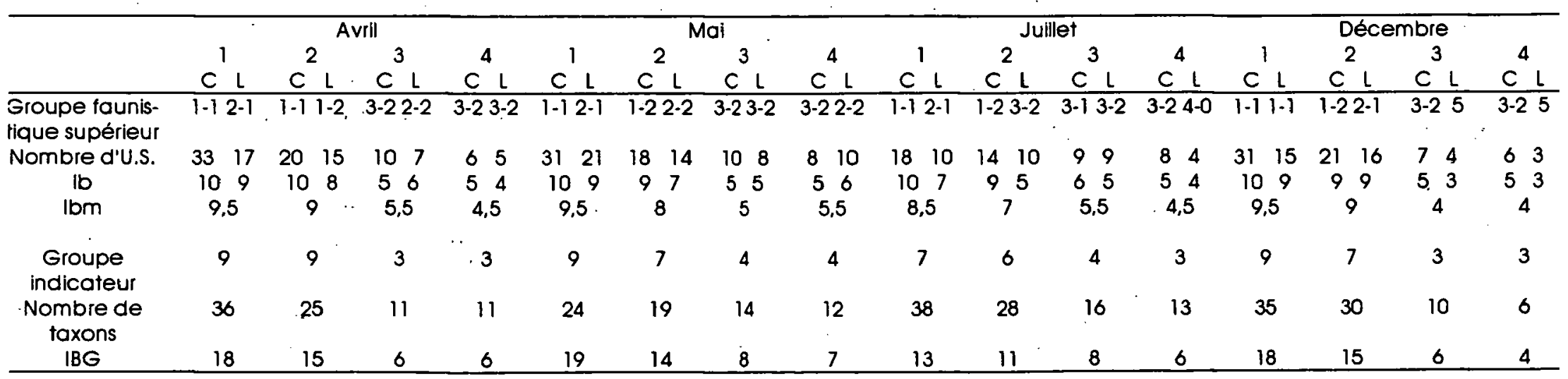



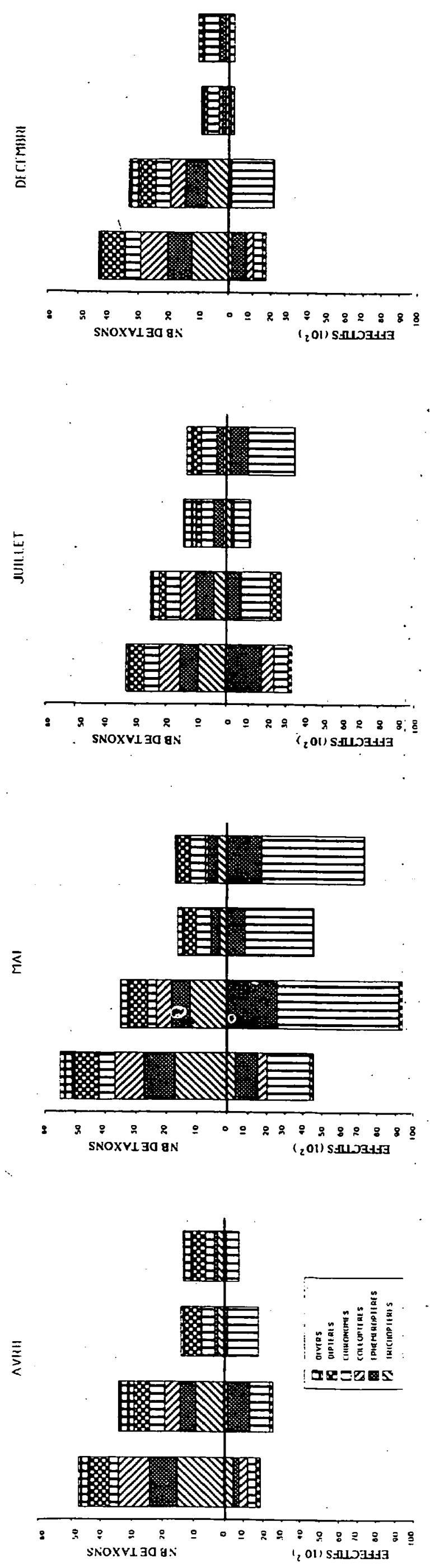

sont observées en fonction des dates d'échantillonnage (Fig. 3), variations le plus souvent en relation avec le cycle de développement. Dans la partie aval, seules 3 espèces de Trichoptères subsistent et une seule, Hydropsyche angustipennis, présente des effectifs relativement élevés, particulièrement en été et en hiver.

L'ordre des Ephéméroptères est représenté par 14 espèces au total. La majorité d'entre elles est localisé dans le cours supérieur de l'Ardières : 12 espèces sur la station de référence, 8 à l'amont de Beaujeu (Fig. 3). Au niveau des stations 3 et 4, trois espèces seulement sont récoltées : Baetis rhodani abondante en mai et juillet, Baetis fuscatus dominante en mai et qui demeure abondante en juillet, enfin Ephemerella ignita bien représentée sur l'ensemble du ruisseau en mai, mais dont les effectifs diminuent fortement en juillet dans le cours inférieur.

Les Coléoptères, appartenant pour la plupart à la famille des Elmidae, sont diversifiés et abondants sur la station de référence où leurs effectifs augmentent sensiblement en juillet (Fig. 3). Ils perdent de leur importance sur le secteur de Beaujeu (station 2) tant en diversité qu'en densité et disparaissent totalement à l'aval.

Les Diptères Chironomidae constituent généralement le groupe d'insectes le plus diversifié dans les milieux d'eau courante. Pour chaque groupe identifié (sous-famille, tribu), d'importantes variations saisonnières des effectifs, en relation avec le grand nombre d'espèces et le caractère d'euryécie et de polluo-résistance de la plupart d'entre elles, sont observées sur l'Ardières (Fig. 3).

Les mollusques, relativement peu diversifiés, disparaissent totalement sur la station 4 ; leur abondance diminue nettement dès l'amont de Beaujeu. Seuls quelques Gastéropodes, Ancylus fluviatilis et Physa acuta, subsistent au niveau de Pré du Bief (station 3):

Les Gammaridae sont abondants sur la station de référence, leur densité diminue fortement sur la station 2 et ils disparaissent de la zone aval.

\section{- Analyse multivariée}

L'étude faunistique a été complétée par une analyse synthétique spatio-temporelle des peuplements de macroinvertébrés benthiques. Cette analyse 
fait appel au logiciel ADDAD ${ }^{1} \mathrm{~d}^{\text {'analyse factorielle }}$ des correspondances (A.F.C.).

Celle-ci a été effectuée à partir d'un tableau de contingence comprenant 16 lignes (dates, stations) et 98 colonnes représentant les variables espèces.

Les abondances des espèces ont été transformées en log 10 afin de minimiser la variabilité inhérente à l'échantillonnage. De plus cette transformation permet d'atténuer le poids des espèces très abondantes et de mieux prendre en compte les espèces moins bien représentées.

Les taxons récoltés une seule fois et représentés par un seul individu ont été éliminés de l'analyse.

Le plan défini par les facteurs F1, F2 (39\% d'inertie) individualise trois groupes de couple station-date : station 1 en avril, mai, décembre (AR11, AR21, AR41) ; station 2 en totalité (AR12 à $A R 42)$ et station 1 en juillet (AR31); stations 3 et 4 (AR13 à AR43), AR14 à AR44) (Fig. 4).

L'axe F1 (28\% d'inertie) oppose la station de référence aux stations 3 et 4 , soumises aux effets des traitements agricoles. La station 2 se trouve dans une position intermédiaire.

Les stations 3 et 4 étant le siège d'une pollution toxique, l'axe 1 peut donc être interprété comme exprimant le gradient de cette pollution. Elouard (1983) dans une étude concernant l'impact des traitements insecticides sur des cours d'eau africains observe le même phénomène.

En ce qui concerne les deux stations non ou peu soumises aux traitements viticoles (st. 1 et 2), l'axe 2 (11,5\% d'inertie) oppose des échantillons d'avril, mai et décembre de la station de référence à ceux de juillet, ainsi qu'à l'ensemble des relevés de la deuxième station. Les analyses physico-chimiques et l'étude faunistique ont mis en évidence la perturbation de nature organique de ce groupe de prélèvements. L'axe 2 peut donc être considéré comme un axe représentant un enrichissement en matières organiques.

Les projections des échantillons des stations 3 et 4 sur l'axe 2 se situent près de celles relatives à la station de référence. La pollution toxique observée sur ce secteur semble donc masquer la pollution de type organique.

1. Association pour la Diffusion et le Développement de l'Analyse des Données.
Le plan défini par les axes F1 et F3 (37 \% d'inertie) oppose sur l'axe F3 les campagnes d'hiver à celles du printemps (Fig. 5). Dans le cas de milieux indemnes de toute pollution ou même sensiblement dégradés, le plan $\mathrm{F} 1 \mathrm{~F} 2$ représente généralement les variations saisonnières de la faune benthique (Perrin 1978, Doledec 1987, Roger et al. 1991). Pour l'Ardières, l'effet pollution qui apparaît sur ce plan met en évidence l'importance de cette perturbation. Une observation similaire a été faite par Musso et al. (1991) sur l'Arc.

Une classification ascendante hiérarchique effectuée sur les 3 premiers axes de l'A.F.C. (47\% de l'inertie) à partir des coordonnées factorielles permet d'établir une répartition des stations et des taxons.

Au niveau des stations, l'existence des deux grands secteurs se trouve confirmée : secteur amont (stations 1 et 2) et un secteur aval (stations 3 et 4 ). Les indices et l'évolution de la richesse taxonomique avaient déjà mis en évidence ces deux secteurs.

Dans le secteur amont le regroupement des prélèvements de juillet au point 1 avec ceux de la deuxième station témoigne de la particularité typologique de la biocénose relevée à cette période sur la station 1 (Fig. 4).

Cette classification permet d'individualiser 4 groupements faunistiques (Fig. 6) établis en fonction de la répartition des taxons sur les différentes stations :

Groupe A : taxons électifs du secteur amont (stations 1 et 2) et dont les effectifs sont généralement plus faibles sur la station 2.

Groupe B : taxons caractéristiques de la station 1.

Groupe C : taxons dominants sur la station 2 .

Groupe $\mathrm{D}$ : taxons communs à toutes les stations.

\section{Interprétation et discussion}

\subsection{Caractéristiques faunistiques des stations}

L'analyse des espèces ou des taxons dont la fréquence et l'abondance sont les plus élevées pour chacun des groupes, conduit à mettre en évidence la particularité faunistique des stations et à établir un diagnostic de qualité des différents secteurs.

\section{- Groupe A}

Dans ce groupe apparaissent deux ensembles de taxons présentant des caractéristiques comparables : 


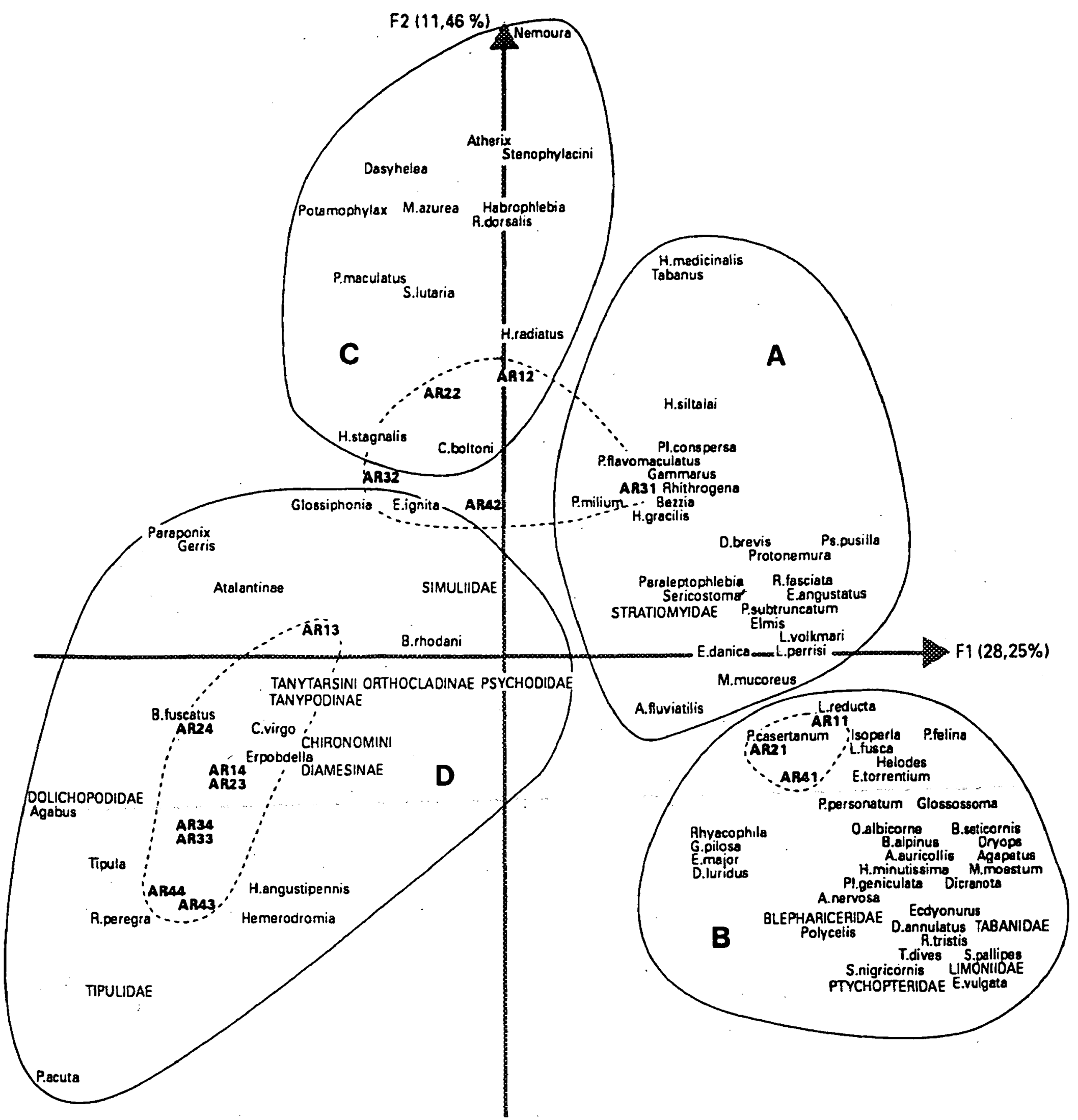

Fig. 4. Distribution des stations-dates et des espèces dans le plan F1F2 de l'analyse factorielle.

Fig. 4. Distribution of the stations-dates and of species in the F1F2 plan of the Factorial Correspondence Analysis.

- Le premier comprend les espèces d'abondance comparable sur les stations 1 et 2 mais dont les effectifs sont faibles en juillet : Rhithrogena sp., Bezzia sp., Pisidium subtruncatum.

Ces variations d'effectifs sont liées au cycle de développement de ces taxons.
- Le deuxième est caractérisé par des taxons dont l'abondance sur la station 2 est toujours plus faible que sur la station de référence.

La forte réduction des effectifs constatée pour les taxons de ce groupe peut être liée à l'augmentation de la charge organique. Sericostoma sp. et 


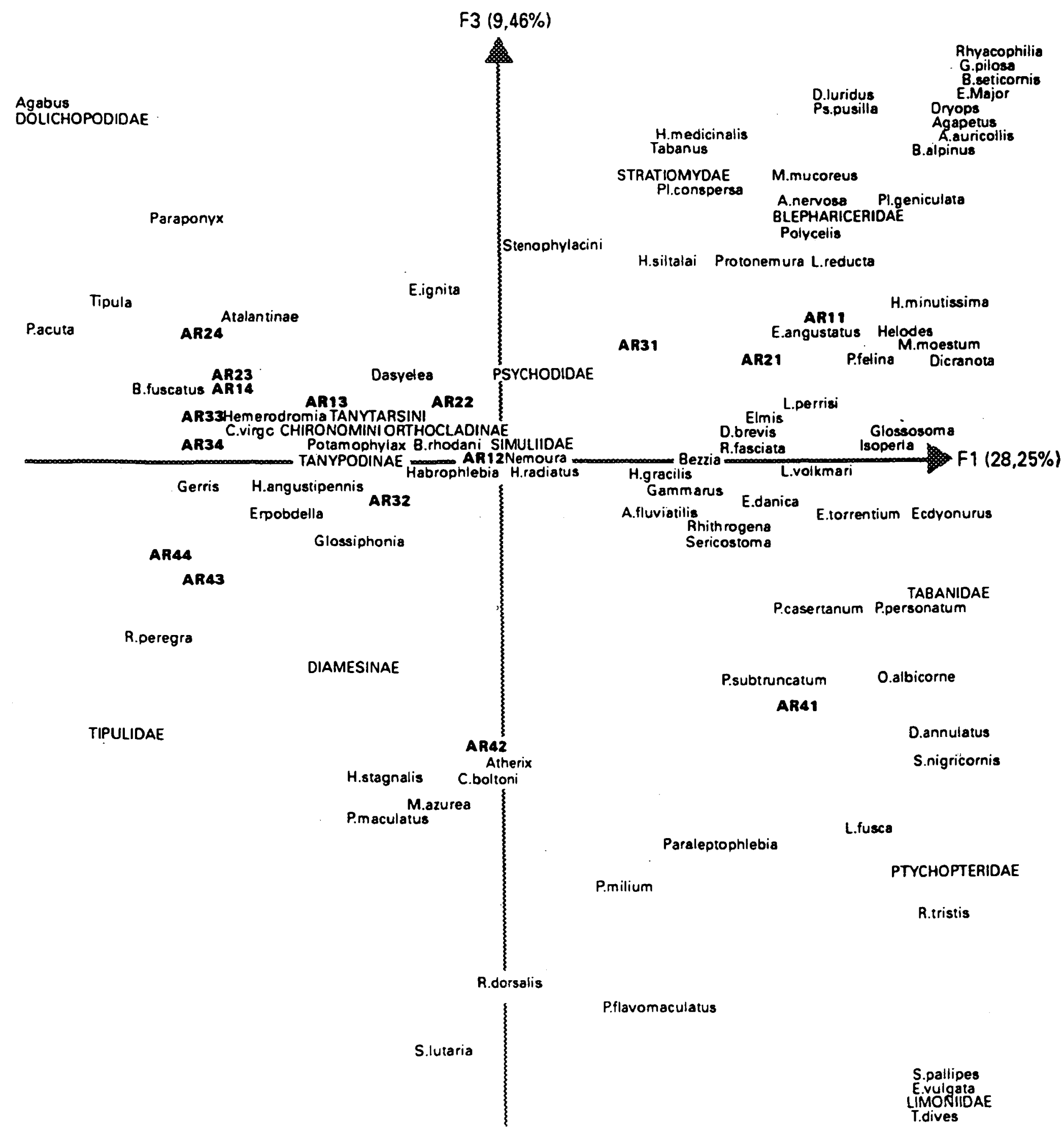

Fig. 5. Distribution des stations-dates et des espèces dans le plan F1F3 de l'analyse factorielle.

Fig. 5. Distribution of the stations-dates and of species in the F1F3 plan of the Factorial Correspondence Analysis.

Melampophylax mucoreus, sont connus pour leur polluosensibilité (Verneaux 1973). Ces espèces, en plus de la baisse d'effectifs sur la station 2, présentent également une plus faible densité en juillet sur la station de référence.
Les Elmidae, généralement considérés comme intolérants à une pollution organique, peuvent cependant résister si le milieu reste bien oxygéné (Learner et al. 1971, 1983). L'évolution de ce peuplement en juillet sur la station 1 , caractérisé par une 


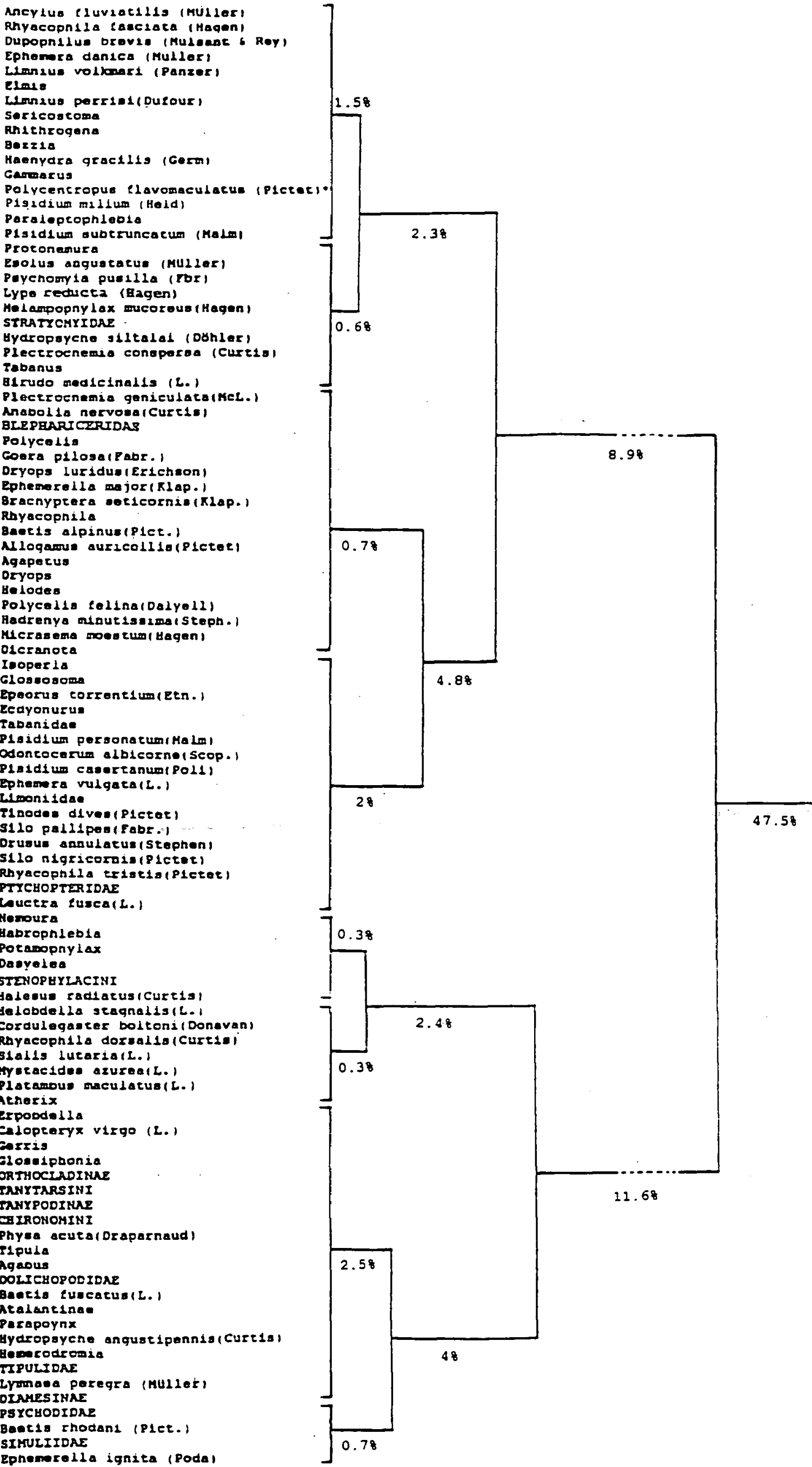

Fig. 6. Classification ascendante hiérarchique des taxons. Pourcentage de l'inertie totale.

Fig. 6. Hierarchical ascending classification of the taxa. Total inert percentage. 
augmentation des effectifs, confirme ce caractère. En revanche, leur diminution sur la station 2 doit être reliée à une perturbation de nature différente, la présence de toxiques n'étant pas à exclure ; ces coléoptères sont très sensibles à une pollution de type industriel même modérée (Young 1961).

Au niveau de la station 2, les baisses d'effectifs d'Ancylus fluviatilis et de Gammarus sp., organismes susceptibles de résister à une charge organique notable, confirment cette observation.

De même Ephemera danica, espèce psammophile ne se développe bien que sur la station 1 au printemps et en hiver. Sa faible densité constatée sur la station 2 ne paraît guère pouvoir être imputée à une pollution de type organique ; cette espèce saprobionte montre qu'un autre type de dégradation existe sur ce secteur.

\section{- Groupe B}

La plupart des espèces de ce groupe est caractéristique des niveaux typologiques supérieurs B2-B3 (Verneaux 1973). Drusus annulatus, Baetis alpinus : B2 ; Silo nigricornis, Rhyacophila tristis : B3 ; ou appartiennent au rhitron : Odontocerum albicorne, Epeorus torrentium (Illies 1968).

La présence de ces espèces associées à la station 1 confirme le niveau typologique théorique calculé pour cette station.

L'absence ou la forte baisse de densité des Heptageniidae (Epeorus torrentium, Ecdyonurus sp.) et de la majorité des Trichoptères au mois de juillet sur cette station 1 n'est pas seulement liée au cycle de développement mais pourrait être également la conséquence d'une augmentation de la charge organique. Ainsi Rhyacophila tristis, très sensible à la présence de phosphates et de nitrates (Moretti \& Mearelli 1980), n'est bien représenté qu'en hiver.

Le cas de Allogamus auricollis est particulier : cette espèce à forte amplitude typologique est caractéristique du niveau typologique B4 (Verneaux 1973). Son absence à partir de juillet sur la station 1 peut être due à son cycle de développement, mais pour la station 2 cette absence permanente semble plus liée à l'existence d'une perturbation de type toxique. En effet Vaillant (1970) a mis en évidence la sensibilité de cette espèce à une pollution industrielle.
Polycelis felina, espèce qui peut proliférer en présence d'une pollution organique mais avec une quantité importante d'oxygène dissous (Macan 1974, Lafont 1975) n'est récoltée que sur la station de référence. Son absence sur les autres secteurs témoigne également de la présence de toxiques.

Ce groupement d'invertébrés met en évidence la particularité typologique de la station 1, sa dégradation en juillet, et autorise la forte présomption de la présence de toxiques sur la station 2 .

- Groupe C

Les espèces de ce groupe sont essentiellement recensées sur la station 2 , quelques-unes d'entre elles (Stenophylax sp., Habrophlebia sp. et Halesus radiatus) se développent également en juillet sur la station de référence.

La plupart de ces taxons est rencontrée tout particulièrement dans les milieux riches en débris organiques grossiers. Ainsi, Halesus radiatus surtout récolté en milieu lénitique est très abondant dans les débris végétaux ; de même, Habrophlebia sp., forme nageuse, rhéobionte se rencontre dans les formations végétales avec forte concentration de débris organiques. Les Diptères, appartenant au genre Dasyhelea vivent dans les amas d'algues dont le développement est le signe d'une charge organique notable.

Ces espèces, inféodées à la station 2 , sont connues comme organismes saprobiontes, elles trouvent sur ce secteur des conditions favorables à leur développement. La majorité d'entre elles présentent d'ailleurs leur plus forte contribution à l'axe F2 de l'AFC.

Elles semblent également susceptibles de résister à la pollution toxique décelée sur cette station.

\section{- Groupe D}

Il englobe 22 taxons ou espèces communes à toutes les stations de l'Ardières. La moitié d'entre eux n'est représentée qu'épisodiquement et/ou par un nombre restreint d'individus.

En revanche, les Chironomidae, les Simuliidae, le genre Hemerodromia (Empididae), trois espèces d'Ephéméroptères (Ephemerella ignita, Baetis fuscatus, Baetis rhodani) et un seul Trichoptère (Hydropsyche angustipennis) sont fréquents et abondants sur l'ensemble du cours d'eau. Ces organismes à caractère euryèce, saprobiontes et polluorésistants présentent dans l'ensemble de fortes contributions aux deux premiers axes. 
Les Simuliidae, organismes filtreurs, sont abondants sur le secteur amont (stations 1 et 2) avec des effectifs plus élevés en juillet. Le développement particulièrement important de ces Diptères confirme l'existence, sur ce secteur, d'une richesse en matières organiques particulaires qui constituent la base de leur nourriture. Au niveau des stations aval, soumises aux effets des traitements viticoles, leur densité diminue notablement et ils disparaissent de ce secteur en décembre. Les produits phytosanitaires employés pour le traitement du vignoble semblent avoir un impact non négligeable sur ces Diptères.

Le genre Hemerodromia se développe plus intensément sur le secteur aval, il est plus abondant et plus fréquent sur la troisième station où il est récolté dans les mousses. C'est une forme carnassière, favorisée par une charge organique qui entraîne le développement de ses proies (Chironomidae, en particulier). Ce taxon semble être résistant à la pollution organique et toxique.

Ephemerella ignita, espèce assez résistante à la pollution organique (Thomas 1981), est surtout récoltée en mai et juillet. Elle est particulièrement abondante en amont de l'Ardières (stations 1 et 2), ses effectifs diminuent fortement en juillet sur la zone aval polluée et cette espèce disparaît même de la station 4 à cette période.

Baetis fuscatus est pratiquement absente de la station 1 ( 5 individus recensés en mai). Cette espèce, caractéristique du niveau $B 7$, présente une forte amplitude typologique (Verneaux 1973). Elle est bien représentée sur les 3 autres points d'étude en mai et juillet, plus particulièrement à Pont de Pizay (station 4).

Les deux espèces Ephemerella ignita et Baetis fuscatus ont des répartitions saisonnières assez semblables.

Baetis rhodani, tolérante à de fortes teneurs en phosphates et nitrates (Leaner et al. 1983), a ses plus fortes densités sur les stations 1 et 2 . C'est au niveau de Pré du Bief (point 3) que les effectifs sont les plus faibles.

Pour ces trois espèces d'Ephéméroptères, l'observation d'une diminution sensible des effectifs au niveau de la station 3 peut révéler une dégradation importante de ce secteur. Cependant $B$. fuscatus semble légèrement moins affecté par ce type de perturbation.
Hydropsyche angustipennis, peu abondant sur le cours supérieur, présente des effectifs plus élevés sur les 2 autres stations, surtout en juillet et décembre. Les larves d'Hydropsychidae sont des organismes filtreurs se nourrissant de particules organiques ou de proies vivantes capturées dans leur filet, elles sont très communes dans les milieux lotiques riches en matière organique particulaire. $H$. angustipennis a la particularité de proliférer en aval des exutoires de lac ou d'étang (Décamps 1968). Les densités élevées, observées sur la partie inférieure de l'Ardières pourraient ainsi être dues aux petites retenues qui jalonnent cette partie du cours d'eau. Cette espèce est également reconnue comme une des plus tolérantes à la pollution organique et à un déficit en oxygène dissous (Wiberg-Larsen 1980). Par ailleurs, la résistance de cette espèce à divers produits toxiques, métaux en particulier $(\mathrm{Cd}, \mathrm{Cu}, \mathrm{Zn})$ démontrée par de nombreux auteurs (Décamps et al. 1973, Besch et al 1977, Zanella 1982, William et al. 1985, Clements et al. 1988) est confirmée dans le cas de l'Ardières, par les effectifs élevés sur la zone aval.

\subsection{Structure des peuplements. Essai de caractéri- sation des types de pollution}

Une biocénose est composée d'une collection d'espèces dont les effectifs résultent des interactions qui s'exercent entre ces espèces et les composantes non biotiques du milieu et entre les espèces elles-mêmes.

Selon Odum (1975) : « Les communautés biotiques naturelles présentent comparativement un petit nombre d'espèces communes représentées par un grand nombre d'individus et un grand nombre d'espèces comptant peu d'individus. "

Toute modification des composantes physiques et chimiques, toute perturbation, entraîne des changements plus ou moins importants dans la composition et la structure des peuplements qui colonisent un milieu donné. Ceci est particulièrement vrai dans le cas de substances toxiques qui peuvent avoir des cibles très spécifiques.

La représentation graphique de la règle d'Odum, applicable en milieu dulcicole, par une courbe richesse taxonomique-abondance, construite à partir de classes d'abondance, donne une courbe caractéristique d'allure sigmoïde (Verneaux 1976).

De telles courbes ont été construites, dans le cas de l'Ardières, par saison et pour chaque station (Fig. 7). La cotation retenue pour les classes 

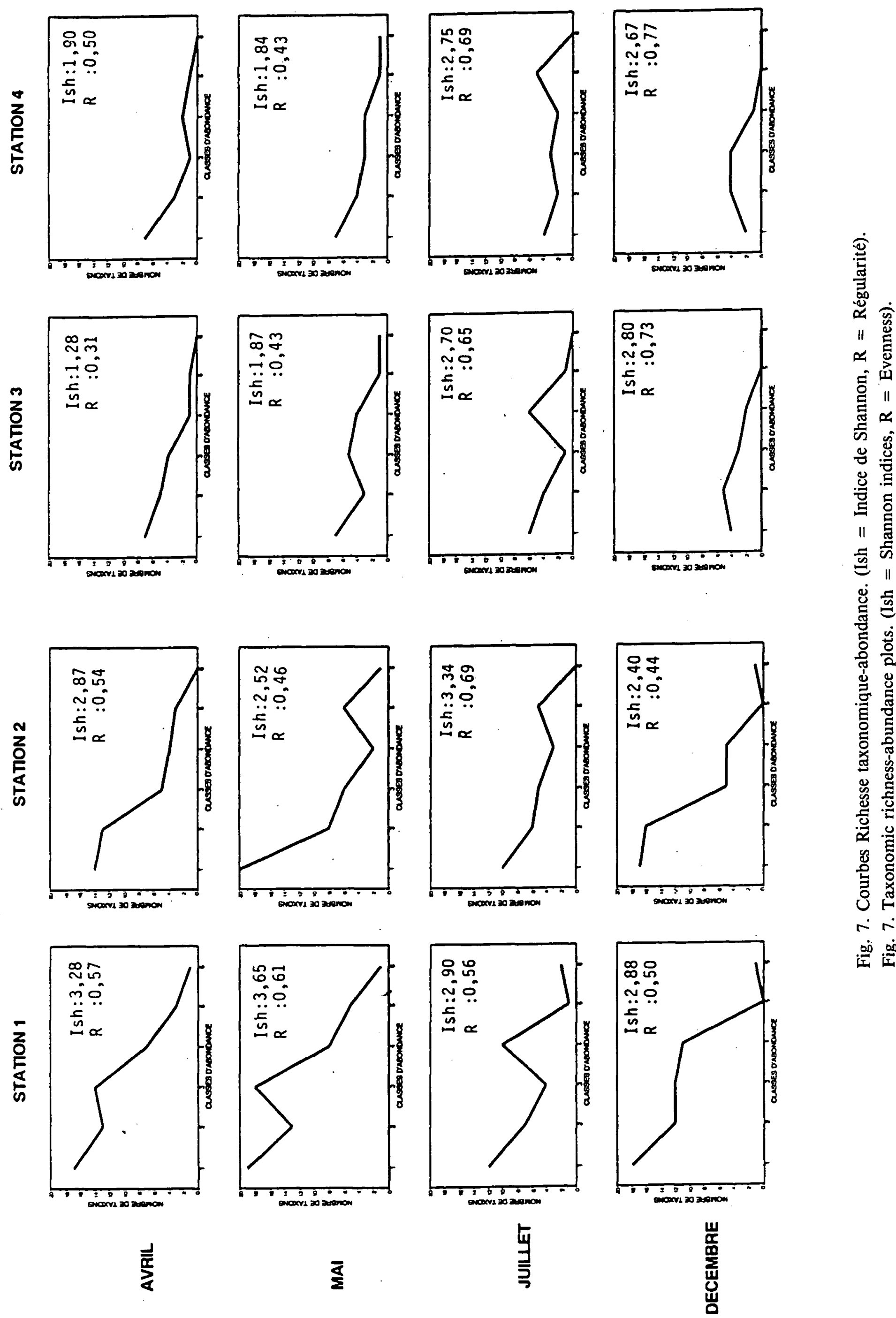

$\bar{\Sigma}$

5
$\exists$
3 
d'abondance est celle proposée par Frontier \& Pichod-Viale (1991). Les bornes des classes suivent une progression géométrique de raison 4.3. Ces classes sont fondées sur des considérations se rapportant à la théorie de l'information. « Une abondance sera significativement supérieure à partir du moment où elle lui est 4,3 fois supérieure. " Frontier \& Pichod-Viale (op. cit.).

A ces courbes ont été associés les indices de diversité de Shannon (Ish) et la régularité (R) (selon Frontier \& Pichod-Viale 1991).

L'intérêt de ces indices étant de permettre la comparaison globale de peuplements appartenant à des sites différents ou d'un peuplement à des saisons différentes.

L'examen des courbes confirme, quelle que soit la période considérée, l'existence de 2 zones distinctes : secteur amont peu perturbé, zone aval fortement polluée.

Si l'on considère les indices obtenus sur les stations 3 et 4 en avril et mai cette observation se trouve vérifiée : les indices de Shannon et les régularités sont nettement inférieurs à ceux calculés sur le secteur amont. La structure des biocénoses est donc très différente.

Les valeurs de ces paramètres peuvent être en relation avec de faibles richesses taxonomiques et des effectifs totaux relativement élevés.

En revanche, sur l'ensemble du secteur aval en juillet et décembre, les indices de Shannon et la régularité sont relativement élevés, en relation avec une richesse taxonomique et des effectifs totaux très réduits. Aucun des taxons ne présentant des effectifs importants, la répartition est homogène.

Les courbes représentant les biocénoses des stations 3 et 4 sont aplaties. Elles témoignent d'une simplification de la structure des communautés. Ce phénomène est vraisemblablement lié à une perturbation de type toxique, particulièrement marquée en décembre peut-être en rapport avec la présence à cette saison de jeunes larves plus sensibles (Buikema \& Benfield 1979, Benfield \& Buikema 1980).

Au niveau de la première station, les courbes, relatives à avril, mai et décembre révèlent une situation subnormale. On observe une légère anomalie au niveau des classes 2 et 3 ; la prépondérance de la classe 3 traduirait l'existence d'une légère pollution organique confirmée par des teneurs élevées en $\mathrm{NO}_{3}{ }^{-}$, en relation avec des apports exogènes pouvant être liés à la nature de l'environnement (zone de pâturage).

En juillet, la courbe montre un déficit des espèces à faibles effectifs (classe 1 à 3). Ce déficit révèle une perturbation en relation avec une augmentation de la charge organique (forte concentration en $\left.\mathrm{PO}_{4}{ }^{--}\right)$mais également imputable au cycle de développement des espèces (émergence de nombreux insectes).

Pour la station 2 au printemps et en hiver, la pente importante de la première partie de la courbe due à un déficit des classes 2,3 et même 4 , traduit une structure biologique plus dégradée que sur la station de référence. Les teneurs élevées en nutrients $\left(\mathrm{NO}_{3}{ }^{-}, \mathrm{NH}_{4}{ }^{+}, \mathrm{PO}_{4}^{---}\right)$. observées sur cette station durant ces périodes confirment la dégradation du secteur. Par ailleurs, en juillet, la courbe apparaît plus aplatie, pouvant révéler, en plus, un syndrome latent de pollution toxique.

Les indices de Shannon en avril et mai sur la première station témoignent d'une bonne qualité du milieu si l'on se réfère aux travaux de Wilhm (1972) qui montre que des indices supérieurs à 3 caractérisent un cours d'eau exempt de pollution.

Sur cette station, en juillet et décembre, ainsi que sur la station 2 (sauf en juillet) on observe des indices et régularités plus faibles. Ces 2 paramètres montrent que sur ce secteur à ces périodes, les structures des biocénoses sont comparables.

En juillet, la station 2 présente un indice de Shannon et une régularité élevés malgré une forte diminution de la richesse taxonomique. Cette structure qui tend vers une équirépartition taxonomique des effectifs est comparable à ce qui est observé sur le secteur aval en juillet et décembre, et témoigne d'une dégradation de type toxique.

Suivant l'allure des courbes, il est possible de caractériser le type de pollution incriminé.

Une courbe en dents de scie est le signe d'une pollution de type organique. Celle-ci entraîne généralement une réduction de la richesse taxonomique : disparition des espèces les plus sensibles, diminution notable des effectifs de certaines espèces moins sensibles et surtout prolifération des organismes saprobiontes favorisés par ce genre de dégradation. 
Lorsqu'il s'agit d'une pollution de nature toxique, cette augmentation d'effectifs ne s'observe jamais ; soit les espèces sont peu sensibles à ces produits et leur abondance ne varie pas, soit au contraire, et c'est généralement le cas, on observe une forte diminution des effectifs de tous les organismes pouvant aller jusqu'à la disparition de nombreuses espèces.

Les effets d'une pollution toxique se traduisent donc par une diminution globale du nombre de taxons et de la densité, la répartition des effectifs en classes d'abondance pouvant être assez régulière et homogène. La courbe richesse taxonomiqueabondance obtenue est alors plus ou moins aplatie et proche de l'axe des abcisses.

\section{Conclusion}

Le bilan général de l'étude met en évidence l'existence de deux secteurs de qualité biologique différente sur ce cours d'eau :

- secteur amont de qualité biologique correcte,

- secteur aval fortement dégradé.

Cependant une analyse biologique détaillée révèle une perturbation de nature organique en été, sur la première station et toute l'année sur la station 2 .

En outre, le comportement particulier de quelques espèces comme Ancylus fluviatilis, Ephemera danica et l'ensemble des Elmidae conduit à soupçonner la présence de toxiques sur cette station.

Dans le secteur aval (stations 3 et 4 ) fortement soumis à une pollution diffuse due aux traitements de la vigne, la faune benthique n'est plus représentée que par quelques formes polluorésistantes.

\section{Remerciements}

Les auteurs remercient $M$. Hervé Pella pour son aide dans les traitements informatiques, $M$. Michel Lafont d'avoir bien voulu critiquer le manuscrit et $\mathbf{M m e} \mathbf{M}$. Taillole qui en a assuré la dactylographie.

Ce travail a bénéficié d'une subvention du Ministère de l'Environnement (SRETIE).

\section{Travaux cités}

Anonyme 1988. - Etude de la contamination par les produits de traitements phytosanitaires sur le bassin versant de l'Ardières. Recherche de solutions adaptées. Rapport Cemagref : $80 \mathrm{p}$.

Benfield E.F. \& Buikema A.L. 1980. - Synthesis of miscellaneous invertebrate toxicity tests. In Aquatic Invertebrate bioassays. ASTM STP 715. A.L. Buikema and J. Cairns Ed. 209 p.
Buikema A.L. \& Benfield E.F. 1979. - Use of macroinvertebrate life history information in toxicity tests. J. Fish Res. Board Can. $36:$ 321-328.

Besch W.K., Schreiber I. \& Herbst D. 1977. - Der Hydrospyche - Toxizitätstest erprobt an fenethcarb. Schweiz. Z. Hydrol. $39: 69-85$.

Cazin B. 1987. - Proposition d'interprétation de résultats d'analyses physico-chimiques. Doc. Cemagref $12 \mathrm{p}$.

Chapman P.M. 1989. - Current approach to developing sediment quality criteria. Environ. Toxicol. Chem. 5 : 957-964.

Chapman P.M. 1991. - Environmental Quality. Criteria. What type should be developing ? Environ. Sci. Technol. 25 (8) : 1353-1358.

Clements W.H., Cherry D.S. \& Cairns J.J. 1988. - Impact of heavy metals on insect communities in streams : a comparison ob observational and experimental results. Can. J. Fish Aquat. Sci. 45 : 2017-2045.

Collet M. 1987. - Enquête sur l'utilisation des produits phytosanitaires sur le bassin versant de la Morcille. Doc. CEMAGREF : $14 \mathrm{p}$.

Cummins K.W. 1975. - The ecology of running waters. Theory and practice. In : Proc. Sandusky river basin symp. Int. Joint. Comm. Int. Ref. Gr. Great Lakes Pollution from land use activities. Ed. D.B. Baher, W.B. Jackson, B.L. Prater : 277-293.

Décamps H. 1968. - Vicariances écologiques chez les Trichoptères des Pyrénées. Annls Limnol. 4 (1) : 1-50.

Décamps H., Besch K.W. \& Vobis H. 1973. - Influence de produits toxiques sur la construction du filet des larves d'Hydropsyche (Insecta, Trichoptèra). C.R. Acad. Sc. Série D $t$ 276 : 375-378.

Doledec S. 1987. - Etude des peuplements de macroinvertébrés benthiques de l'Ardèche dans son cours inférieur. Arch. Hydrobiol., 109 : 541-565.

Elouard J.M. 1983. - Impact d'un insecticide organophosphoré, le Téméphos sur les entomocoenoses associées aux stades préimaginaux du complexe Simulium damnosum. Th. (Diptera, Dimuliidae). Thèse Doct. Etat, Paris Sud : $571 \mathrm{p}$.

Frontier S. \& Pichod-Viale D. 1991. - Ecosystemes. Structurefonctionnement-évolution. Masson Ed. Paris : 392 p.

Illies J. 1968. - Ephemeroptera (Eintagfliegen). Handb. Zool. 4 (2) $2 / 5: 1-63$.

Kimball K.D. \& Levin S.A. 1985. - Limitations of Laboratory bioassays : the need for ecosystem-level testing. Bioscience, 35 (3) : 165-171.

Lafont M. 1975. - Incidences écologiques des rejets de stations de sport d'hiver sur les cours d'eau d'altitude. Etude C.T.G.R.E.F., $n^{\circ} 6: 52 \mathrm{p}$.

Lapoint T.W., Melancon \& Morris M.K. 1984. - Relationships among observed metal concentrations criteria, and benthic community structural responses in 15 streams. J. WPCF, 56 (9) : 1030-1038.

Leaner M.A., Williams R., Harcup M. \& Hughes B.D. 1971. A survey of the macro-fauna of the River Cynon, a polluted tributary of the River Taff (South Wales). Freshwat. Biol. $1: 339-367$.

Leaner M.A., Densen J.W. \& Illes T.C. 1983. - A comparison of some classification methods used to determine benthic macro-invertebrates species associations in river survey work based on data obtained from river Ely, South Wales. Freshwat. Biol. 13 : 13-36. 
Macan T.T. 1974. - Running water. Mitt. Internat. Verein. Limnol. $20: 301-321$.

Malavoi J.R. \& Souchon Y. 1989. - Méthodologie de description et quantification des variables morphodynamiques d'un cours d'eau à fond caillouteux. Exemple d'une station sur la Filière (Haute-Savoie). Rev. Géog. Lyon, 64 (4) : 252-259.

Moretti G.P. \& Mearelli M. 1980. - Ecological profiles in three Rhyacophila species. In : Proc. of the 3rd Inst. Symp. on Trichoptera. Ed. by G.P. Moretti. Série Entomologica. Vol. 20 : 472 p.

Munoz J.F. \& Belamie R. 1990. - Identification et comportement des produits utilisés en viticulture. Etude d'un bassin versant de l'Ardières (Beaujolais). $\mathrm{XX}^{\mathrm{e}}$ Congrès « groupe français des pesticides » $: 6 \mathrm{p}$.

Musso J.J., Prévost G., Légier P. \& Playoust C. 1991. Contribution à la connaissance des cours d'eau méditerranéens de basse altitude : le réseau hydrographique de l'Arc (Bouches du Rhône, France). Etat des peuplements en référence aux perturbations anthropiques. Annls Limnol. 27 (1) : 75-85.

Nisbet M. \& Verneaux J. 1970. - Composantes chimiques des eaux courantes. Discussion et proposition de classes en tant que bases d'interprétation des analyses chimiques. Annls Limnol. 6 (2) : 161-190.

Nore V. 1988. - Caractérisation et évolution des activités humaines sur le bassin versant de l'Ardières (Rhône Beaujolais), érosion des sols, dynamique de l'écoulement et du ruissellement sur le sous-bassin versant de la Morcille (Villé Morgon, Rhône). D.E.S.S. "Aménagement et Développement Rural ". Université Lumière, Lyon II : $52 \mathrm{p}+$ annexes.

Odum E.P. 1975. - Ecology. Second Ed. H. Rinehart and Winston, London : $244 \mathrm{p}$.

Odum E.P. 1984. - The Mesocosm. Bio Science. 34 (9) : 558-562.

Perrin J.F. 1978. - Signification écologique des peuplements benthiques du Haut-Rhône français. Thèse Doct. Spécialité, Lyon : $171 \mathrm{p}$.

Roger M.C., Faessel B. \& Lafont M. 1991. - Impact thermique des effluents du Centre de Production Nucléaire du Bugey sur les invertébrés benthiques du Rhône. Hydroécol. Appl. $1: 63-110$.

Roulier J.L. 1968. — Mobilité et rémanence des métaux utilisés dans les traitements phytosanitaires de la vigne. Rapport DEA. Cas du bassin versant de la Morcille (Beaujolais). INSA Lyon. $45 \mathrm{p}$.

Thomas A.G.B. 1981. - Travaux sur la taxonomie, la biologie et l'écologie d'insectes torrenticoles du sud-ouest de la France (Ephéméroptères et Diptères ; Dixidae, Cecidomyiidae, Rhagionidae et Athericidae) avec quelques exemples de perturbations par l'homme. Thèse Doct. Etat, Toulouse : $330 \mathrm{p}$.
Tuffery G. \& Verneaux J. 1967. — Méthode de détermination de la qualité biologique des eaux courantes. Exploitation codifiée des inventaires de la faune de fond. Trav. Div. Qual. Eaux P. Pisci. C.T.G.R.E.F. Paris : 23 p.

Vaillant F. 1970. - Transformation de la faune de l'Isère à Grenoble sous l'effet de la pollution. Trav. Lab. Hydrobiol. 61 : 17-37.

Verneaux J. 1973. - Cours d'eau de Franche Comté (Massif du Jura). Recherches écologiques sur le réseau hydrographique du Doubs. Essai de biotypologie. Thèse Doct. Etat, Besançon : $260 \mathrm{p}$.

Verneaux J. 1976. - Fondements biologiques et écologiques de l'étude de la qualité des eaux continentales. Principales méthodes biologiques. In Pesson : La pollution des eaux continentales. Gauthier Villars Ed. : 312 p.

Verneaux J., Galmiche P., Janier F. \& A. Monnot. 1982. - Une nouvelle méthode pratique d'évaluation de la qualité des eaux courantes. Un indice biologique de qualité générale (IBG). Ann. Sci. Univ. Franche Comté, Besançon. $4^{\mathrm{e}}$ série, 3 : 11-21. (Norme AFNOR T $95 \mathrm{~F}$ doc 17, Mai 1985).

Wallace J.B., Huryn A.D., Lugthard G.J. 1991. - Colonization of a headwater stream during three years of seasonal insecticidal applications. Hydrobiologia, 211 : 65-76.

Washington H.G. 1984. - Diversity, biotic and similarity indices. A review with special relevance to aquatic ecosystems. Water Res. 18 : 653-694.

Wiberg-Larsen P. 1980. - Key to larvae of the Danish Hydropsychidae (Trichoptera) with notes on distribution and ecology. Ent. Meddr. 47 : 125-140.

Wilhm J.L. 1972. - Graphic and mathematical analyses of biotic communities in polluted streams. Ann. Ress. Ent. 17 : 223-252.

Williams K.A., Green D.W.J. \& Pascoe D. 1985. - Studies on the acute toxicity of polluants to freshwater macroinvertebrates. 1. Cadmium. Arch. Hydrobiol. 102 (4) : 461-471.

Winner R.W., Boesel M.W. \& Farnell 1980. - Insect community structure as an index of heavy-metal pollution in lotic ecosystems. Can. J. Fish. Aquat. Sci. 37 : 647-655.

Young F.N. 1961 . - Effects of pollution on natural association of water beetles. Purdue Univ. Engineering Bull., Engineering séries 106. 15 (2) : 373-380.

Zanella E.F. 1982. - Shifts in caddisfly species composition in Sacramento River Invertebrates. Communities in the presence of heavy metal contamination. Bull. Environm. Contam. Toxicol. 29 : 306-312. 